\title{
Endogenous Attention Signals Evoked by Threshold Contrast Detection in Human Superior Colliculus
}

\author{
Sucharit Katyal and David Ress \\ Departments of Neuroscience and Psychology, The University of Texas at Austin, Austin, Texas 78712
}

Human superior colliculus (SC) responds in a retinotopically selective manner when attention is deployed on a high-contrast visual stimulus using a discrimination task. To further elucidate the role of SC in endogenous visual attention, high-resolution fMRI was used to demonstrate that $\mathrm{SC}$ also exhibits a retinotopically selective response for covert attention in the absence of significant visual stimulation using a threshold-contrast detection task. SC neurons have a laminar organization according to their function, with visually responsive neurons present in the superficial layers and visuomotor neurons in the intermediate layers. The results show that the response evoked by the threshold-contrast detection task is significantly deeper than the response evoked by the high-contrast speed discrimination task, reflecting a functional dissociation of the attentional enhancement of visuomotor and visual neurons, respectively. Such a functional dissociation of attention within SC laminae provides a subcortical basis for the oculomotor theory of attention.

Key words: contrast detection; optic tectum; superior colliculus; attention; fMRI; midbrain

\section{Introduction}

The superior colliculus (SC) is a laminated structure: superficial layers contain neurons that respond to visual stimulation (visual neurons), and intermediate layers contain neurons that discharge before saccadic eye movements, as well as visual stimulation (visuomotor neurons). Electrophysiology studies in macaque have shown correlates of attention in both visual and visuomotor neurons (Wurtz and Mohler, 1976; Ignashchenkova et al., 2004; Cavanaugh et al., 2006). Ignashchenkova et al. (2004) measured single-unit neuronal activity and showed that an exogenous cue enhanced the stimulus-evoked activity of the visual and visuomotor neurons, but only the visuomotor neurons also exhibited a baseline enhancement of activity for the delay period between the cue and stimulus. This baseline visuomotor activity was proposed to be a correlate of the oculomotor theory of attention (Rizzolatti et al., 1987; Moore et al., 2003). Moreover, an endogenous cue did not evoke a significant baseline enhancement, suggesting that the visuomotor neurons may not be modulated by endogenous attention.

In contrast, other monkey studies have shown endogenous attention effects in SC intermediate layer neurons (Kustov and Robinson, 1996; Fecteau et al., 2004). Recent work has also demonstrated that the modulation of SC activity in monkeys by mi-

\footnotetext{
Received July 9, 2013; revised Nov. 6, 2013; accepted Nov. 28, 2013.

Author contributions: S.K. and D.R. designed research; S.K. performed research; S.K. analyzed data; S.K. and D.R. wrote the paper.

This work was supported by NSF BCS 1063774 . We thank Gary Glover for providing the spiral acquisition fMRI pulse sequence, and Jeff Luci and the members of Alex Huk's laboratory who assisted in the creation of the MRIcompatible LCD display. We thank Eyal Seidemann, Alex Huk, Russ Poldrack, and Mike Beauchamp for helpful comments regarding the work presented here.

Correspondence should be addressed to Dr David Ress, The University of Texas at Austin, 1 University Station, C7000, NHB 3.366, Austin, TX 78712. E-mail: ress@utexas.edu.

DOI:10.1523/JNEUROSCI.3026-13.2014

Copyright $\odot 2014$ the authors $\quad 0270-6474 / 14 / 340892-09 \$ 15.00 / 0$
}

crostimulation and pharmacology directly affects behavior in visual tasks, suggesting a role for SC in endogenous attention (Cavanaugh and Wurtz, 2004; Müller et al., 2005; Cavanaugh et al., 2006; Lovejoy and Krauzlis, 2010; Zénon and Krauzlis, 2012). Some of these studies report that this behavioral modulation is specific to the intermediate and not superficial layers (Müller et al., 2005; Cavanaugh et al., 2006).

In human visual cortex, the presence of endogenous attention signals was demonstrated by an fMRI response for attention in the absence of external visual stimulation (Kastner et al., 1999). This endogenous attention response was also shown using an event-related threshold contrast detection paradigm on trials where no stimulus was present (Ress et al., 2000), and was labeled as an fMRI "base response" of attention (analogous to baseline enhancement of neuronal firing rate).

In a previous study, we used high-resolution fMRI to show that covert attention toward a consistently present high-contrast visual stimulus using a speed-discrimination task boosts responses close to the superficial SC surface, whereas responses evoked by high-contrast stimulus alternation protruded more deeply into SC (Katyal et al., 2010). This superficial response profile evoked by pure attention could have been due to an enhancement of the visual neurons, or both the visual and visuomotor neurons; our fMRI measurements could not resolve this fine-grained spatial distinction.

In this study, we measured the attentional base response in SC during a threshold-contrast detection task using a blocked paradigm. A significant response in the detection task was evident, so we compared its depth profile with that of the response evoked by the discrimination task (Katyal et al., 2010). The use of threshold-contrast stimuli was expected to greatly reduce the response of superficial visual neurons, allowing us to test the hypothesis that the detection response in SC is mediated by intermediate layer neurons. The detection response was indeed found to occur 

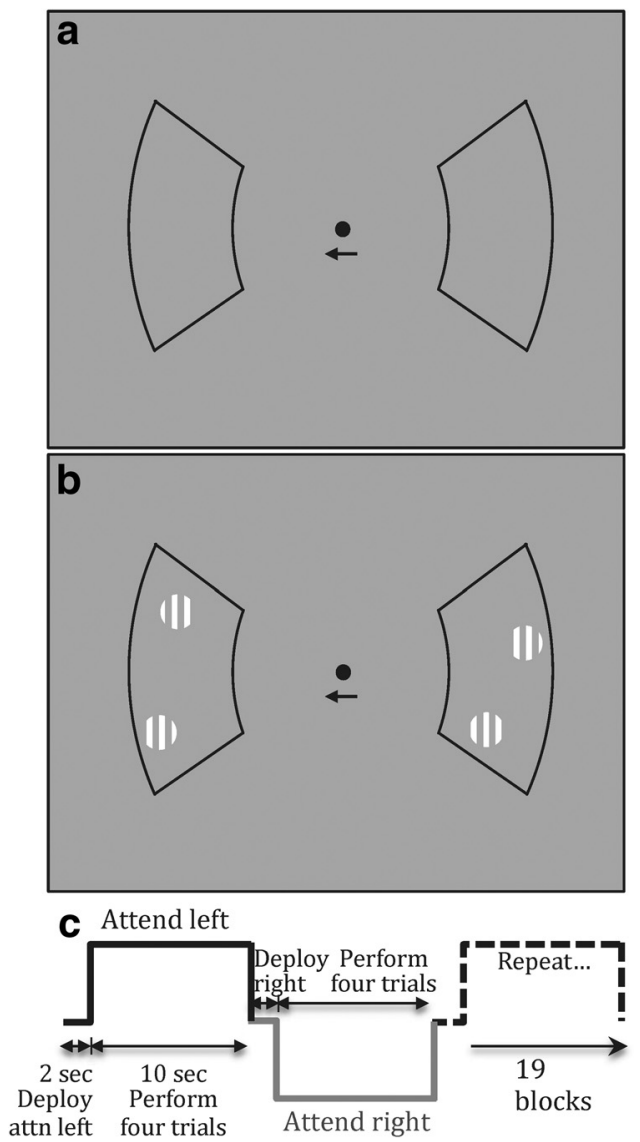

Figure 1. Stimulus and task for detection experiments: $\boldsymbol{a}$, blank sectors; $\boldsymbol{b}$, sectors with Gabor detection targets; $c$, subjects alternated their attention between left and right sectors. Blocks began with a $2 \mathrm{~s}$ delay to deploy attention upon the cued sector, and then subjects performed four $2.5 \mathrm{~s}$ trials. During each trial, there was a $50 \%$ probability that two thresholdcontrast Gabors would be presented briefly at a random time and location within each sector. At the end of each trial, subjects pressed a button to indicate their yes/no detection judgment.

more deeply than the discrimination response, providing new evidence that endogenous attention is linked to the activity of the SC oculomotor circuitry in the human brain.

\section{Materials and Methods}

\section{Subjects}

Five male subjects participated in two to four, $2 \mathrm{~h}$ long scanning sessions: one localizer to characterize the representation of the stimulus aperture, 1-3 contrast-detection sessions, and 1-3 speed-discrimination sessions. Three subjects also performed a control-experiment session. All sessions consisted of $17-18,228 \mathrm{~s}$ runs. The first $12 \mathrm{~s}$ of data were discarded to reduce transients. All subjects had normal or corrected-to-normal visual acuity. Informed consent was obtained from all subjects.

\section{Stimulus protocols}

Stimuli were generated using MATLAB (MathWorks) running PsychToolbox-3 (Brainard, 1997; Pelli, 1997) on a Macintosh Pro computer. Stimuli were presented using a shielded, MRI-compatible 43-inch LCD television located $2.8 \mathrm{~m}$ from the subject's eyes, and viewed through a mirror placed on the head-coil. The display was calibrated both for gamma variations, and to ensure a spatial flat field. In two sessions, an equipment problem required that we use a back-projection display, which was calibrated psychophysically (see Threshold-contrast detection, below).

Threshold-contrast detection. Two black-outlined $45^{\circ}$-width sectors were located along the horizontal meridian to the left and right of the central fixation dot at eccentricity $4^{\circ}-7.5^{\circ}$ (Fig. 1a). Subjects alternated attention between the two sectors in $12 \mathrm{~s}$ blocks. Each alternation began with a $2 \mathrm{~s}$ duration fixation dot color-change, cueing the subject to attend to the left sector (Fig. 1c). For the remainder of the block, a small arrow below fixation indicated the attended sector. The remaining $10 \mathrm{~s}$ contained four trials. For the first $2 \mathrm{~s}$ of each trial there was a $50 \%$ probability of occurrence of a pair of small Gabors $\left(0.25^{\circ} 2 \sigma\right.$ diameter $)$ briefly presented $(\sim 100 \mathrm{~ms})$ at a random time and location, independently in each of the two sectors (Fig. 1b). The black outline clearly demarcated the appropriate aperture for the cued attention. The brief presentation and random positioning of the Gabors made the task more difficult, enabling the use of higher-contrast stimuli that could be accurately rendered with our LCD display system. At the end of the $2 \mathrm{~s}$ stimulus period, the fixation dot turned yellow for $0.5 \mathrm{~s}$, cueing the subject to indicate their judgment about the presence or absence of the Gabors in the attended sector by pressing one of two buttons with their right hand. After four trials on the left side, the fixation changed color for two seconds, cueing subjects to attend to the right sector, where they then performed the same task. This attentional alternation proceeded for 19 blocks, creating runs lasting $228 \mathrm{~s}$.

In two sessions (localizer and contrast-detection repeat for Subject 1) we used a back-projection display. Such displays cannot be accurately calibrated because they produce a strong scattered-light component. We therefore obtained contrast thresholds psychophysically using the backprojected stimulus within the scanner bore. The measured threshold contrast value for these two sessions was substantially higher $(16 \%$ compared with $6.5 \%$ for the LCD), very likely reflecting the display's lower contrast performance.

Localizer. A sector of moving dots (4\% speed) was presented at the same aperture as the contrast-detection stimulus in $12 \mathrm{~s}$ alternating blocks on the left and right sides respectively to delineate retinotopic regions-of-interest (ROIs). The sector was subdivided into $2 \times 2$ virtual sections; each section was a smaller sector located at two different eccentricities and polar angles. During each $2 \mathrm{~s}$ trial, dots in one section moved faster or slower than all other sections. Subjects' task was to fixate while discriminating dot speed, and then indicate their judgment by pressing a button at the end of the trial. There were 19 alternations of the movingdot stimulus. Task performance of the subjects was maintained at $\sim 71 \%$ by adjusting the magnitude of the speed difference between dots using a pair of randomly interleaved two-up-one-down staircases.

Threshold-contrast control. Stimulus was similar to the contrast detection experiment with two black-outlined sectors $\left(45^{\circ}\right.$ polar angle, $4^{\circ}-7.5^{\circ}$ eccentricity) along the horizontal meridian on each side of the fixation dot. Instead of the presentation of Gabor stimuli being counter-balanced within the two sectors for each block, Gabors were presented in a $12 \mathrm{~s}$ blocked-alternation between the left and right sectors to maximize any possible fMRI response contrast evoked by the faint stimuli. The presentation frequency, duration, and contrast of the Gabors were matched with the contrast-detection experiment for each subject. There were 19 blocks of stimulus alternation. Attention was directed away from the stimulus apertures by having the subjects perform a demanding task at fixation. The fixation dot changed colors rapidly (every $300 \mathrm{~ms}$ ), and subjects were required to respond every time the color became green by pressing a button within a $2 \mathrm{~s}$ response window.

Speed discrimination. Two sectors of moving dots ( $4 \%$ s speed) were presented along the left and right horizontal meridians (eccentricity $2^{\circ}-$ $11^{\circ}$, polar angle $144^{\circ}$ ) simultaneously (Katyal et al., 2010). These sectors appeared consistently on each trial, so that the visual stimulus component of the task did not vary. The sectors were divided into $2 \times 4$ virtual sections (two different eccentricities and four different polar angles), and the task of the subjects was, once again, to discriminate whether the dots in one of the sections were moving faster or slower than dots in other sections. The spatial uncertainty of the single section with a different speed encouraged subjects to distribute their attention over the entire sector. A small arrow below the fixation dot cued subjects to alternate their attention between the left and right sectors in $12 \mathrm{~s}$ blocks. Trials had a $2 \mathrm{~s}$ duration with subjects responding within the last $0.5 \mathrm{~s}$. Performance was again maintained with the staircase procedure described above.

\section{MRI methods}

Imaging was performed on a 3T scanner (Signa Excite HD, GE Healthcare) using the GE-supplied eight-channel head coil. Eight 1.2-mm-thick 
Table 1. (1) Threshold-contrast values, (2) spatial centroid difference between the detection response and retinotopic R0Is, and (3) mean and 68\% Cls for the difference between temporal onsets of the response between left and right colliculi

\begin{tabular}{|c|c|c|c|c|c|}
\hline \multirow[b]{2}{*}{ Subject } & \multirow[b]{2}{*}{ Threshold contrast (\%) } & \multicolumn{2}{|c|}{ Spatial centroid difference (mm) } & \multicolumn{2}{|c|}{ Intercollicular delay difference (s) } \\
\hline & & Left & Right & Mean & $68 \% \mathrm{Cl}$ \\
\hline 1 & 6.5 & 0.69 & 0.68 & 7.7 & $5.4-9.8$ \\
\hline 2 & 9.5 & 1.06 & 0.66 & 14.6 & $12.8-16.06$ \\
\hline 3 & 4.5 & 0.56 & 1.13 & 8.6 & $5.6-10.8$ \\
\hline 4 & 6.5 & 0.39 & 0.77 & 11.5 & $9.1-13.7$ \\
\hline 5 & 8.0 & 1.62 & 0.40 & 4.9 & $0.6-8.5$ \\
\hline Mean & 7.0 & 0.86 & 0.72 & 9.5 & $4.9-14.2$ \\
\hline
\end{tabular}

Individual data is shown for all subjects and the means across subjects.

quasi-axial slices (170 mm field-of-view) covered the SC with the prescription oriented approximately perpendicular to the neuraxis. A set of T1-weighted structural images was obtained on the same prescription at the end of the session using a three-dimensional (3D) spoiled gradient echo (SPGR) sequence. These images were used to align the functional data to a segmented reference volume anatomy.

Functional images were obtained on a prescription coaligned with the preceding structural images. A $6.4 \mathrm{~ms}$ windowed-sinc pulse was used to provide a sharp slice-select resolution. We used a three-shot outwardspiral acquisition (Glover and Lai, 1998) to obtain an in-plane pixel size of $1.2 \mathrm{~mm}\left(T_{E}=40 \mathrm{~ms} ; T_{R}=1 \mathrm{~s}\right.$, with three shots, volume acquired every $3 \mathrm{~s}$; Katyal et al., 2012).

The structural images collected in each session were used to align the functional data to a 3D reference volume, which was acquired for each subject in a separate session. The reference volume was T1-weighted with good tissue-CSF contrast, acquired using a 3D, inversion-prepared, fastSPGR sequence (minimum $T_{E}$ and $T_{R}, T_{I}=450 \mathrm{~ms}, 15^{\circ}$ flip angle, isometric voxel size of $0.7 \mathrm{~mm}$ ).

\section{Image analysis}

Analysis of the fMRI data was performed using the mrVista software package (http://white.stanford.edu/newlm/index.php/MrVista) and additional tools developed in our lab within the mrVista framework. Preprocessing included motion and timing corrections, as well as spatial and temporal normalization; no spatial or temporal smoothing was performed (Katyal et al., 2012).

Surface-based analysis. We segmented the brainstem tissue using a combination of automatic and manual methods provided by the ITK-SNAP application (Yushkevich et al., 2006). A smooth but accurate surface was then fit to the CSF-tissue interface of the SC using a deformable-surface algorithm (Xu et al., 2006). This surface provided vertices and outward normal vectors used as a reference for the laminar calculations described below as well as a means to visualize the functional data. A nearestneighbor distance map was calculated between the SC tissue voxels and the vertices of the SC surface to measure depth $(s)$ in the reference volume. Functional data were then aligned and resampled to the reference volume (Nestares and Heeger, 2000).

Based on the distance mapping of the voxels, fMRI time-series data were averaged over $0.5-2.2 \mathrm{~mm}$ in depth from SC surface for the contrast detection and control experiments, $0-1.8 \mathrm{~mm}$ for the attention with stimulation experiment and $0-1.6 \mathrm{~mm}$ for the retinotopic localizer session. The depth ranges were chosen to yield the most reliable response based on the number of significantly active voxels at a threshold of $p<$ 0.05 (see below). Results were similar, but somewhat weaker, when different depth ranges (e.g., $0-2,1-3 \mathrm{~mm}$, etc.) were used in the analysis of these experiments. A sinusoid at the stimulus repetition frequency was then fit as a model to the mean across runs of the depth-averaged time series to obtain response amplitude. In standard Fourier-domain temporal analysis, these fits can also measure phase and coherence. The phase measures the time delay of the response relative to the onset of the lefthemifield task blocks. We used standard Fourier-domain analysis for our Localizer responses to obtain a measure of the hemodynamic delay. We used this delay to analyze the data from our experimental attention and control conditions.

Nonparametric statistical testing. The noise in fMRI data is known to have a non-Gaussian distribution (Biswal et al., 1995; Holmes et al., 1997;
Glover et al., 2000; Krüger and Glover, 2001). Typically, fMRI data are therefore "whitened" by performing a mixture of spatial and temporal blurring (Worsley and Friston, 1995; Friston et al., 2000). To maintain our high spatial resolution, we wanted to avoid any blurring. Therefore, we used nonparametric methods to estimate statistical significance. Significance was estimated for each voxel by bootstrapping: resampling the time-series from the many individual runs $(\geq 18)$ with replacement, taking their average, and fitting it with the model sinusoid from the localizer to obtain an amplitude; this procedure was repeated 10,000 times. The $p$ value was then estimated as the fraction of resampled amplitudes less than zero for a positive value of the mean amplitude, and greater than zero for a negative value.

ROI generation. Using the localizer sessions ROIs were created that surrounded the peak depth-averaged amplitude on each colliculus. Starting from this peak location, a $p$ value threshold was gradually increased until a target size $\left(\sim 4.5 \mathrm{~mm}^{2}\right)$ of contiguous voxels was included in the ROI. Target size was chosen based on the expected retinotopic representation of the stimulus aperture, based on previous retinotopy measurements (Katyal et al., 2010). All analyses for the detection, control, and discrimination experiments were performed within these independently localized ROIs.

A similar procedure was also used to define ROIs for the detection experiment. The distances between the spatial centroids of the ROIs obtained from the localizer and detection experiments were used as a measure of spatial localization (Table 1).

Response amplitude calculation. Response amplitudes were modeled using two approaches. In the more conservative of the two approaches, the depth averaged time-series data from the detection, discrimination and control experiments were fit with a sinusoid at the stimulus alternation frequency whose time delay (phase) was determined by the mean phase of the right SC ROI from the localizer session (use of the mean phase from the left ROI data had a very similar spatial pattern with signs reversed). The approach forced the data to vary at the time delays obtained in the localizer experiment, which were very generally very nearly counter-phased. Amplitudes were also calculated using a more liberal approach, in which sinusoids at the stimulus alternation frequency with the mean phase of the right ROI of that session. This permitted the time delay between the colliculi to vary freely from session-to-session.

\section{Depth profile analysis}

Profiles were calculated within the ROIs obtained from the localizer session. The superficial coordinates of these ROIs were extended inward and outward along the surface normals obtained from the surface model. Within the extended ROIs, we took the complex Fourier-domain response (amplitude and phase) as a function of depth and convolved a boxcar-smoothing kernel $(0.7 \mathrm{~mm}$ width) as a function of depth; the amplitude of this convolution was the depth profile, $A(s)$ (Ress et al., 2007; Khan et al., 2011). We again bootstrapped, $>10,000$ iterations, the depth complex responses to obtain confidence intervals (CIs) on the depth amplitude profiles. Depth profiles were calculated for both the detection and discrimination experimental conditions for each subject, and these profiles were then averaged across subjects. The final subjectaverage profiles were then normalized by their maximum response to permit evaluation of response components between layers.

The fMRI response is mediated by a blood oxygen-level-dependent (BOLD) signal. The spatial specificity of BOLD response at $3 \mathrm{~T}$ is limited 

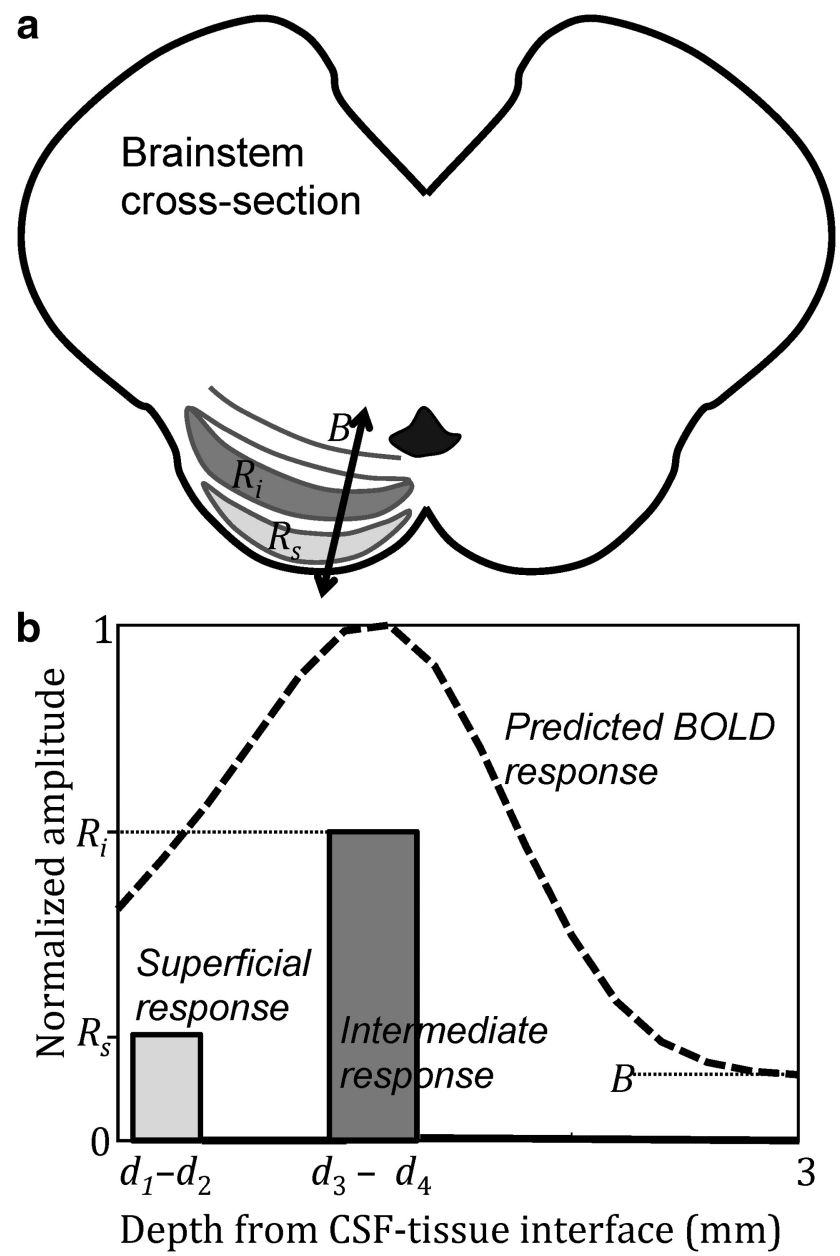

Figure 2. Depth model of the SC BOLD response. $\boldsymbol{a}$, A schematic of the SC layers and the model, which consists of responses in the superficial $\left(R_{s}\right)$ and intermediate $\left(R_{i}\right)$ gray layers, together with a nonspecific response that extends throughout the SC and into the CSF $(B) . \boldsymbol{b}$, Example response components (solid line) and the simulated depth profile (dashed line).

to $\sim 1 \mathrm{~mm}$ full-width at half maximum (FWHM; Duong et al., 2001; Thompson et al., 2005) and also by our $1.2 \mathrm{~mm}$ voxel sampling. Accordingly, we obtained depth information from two global moments of the distribution. First, we calculated the location of the peak amplitude in these profiles between conditions, and second, we calculated spatial centroids: $c=\int_{0}^{d_{m}} s A(s) d s / \int_{0}^{d_{m}} A(s) d s$, where $d_{m}$ is the maximum depth of the calculation, which we chose to be either 2.5 or $3 \mathrm{~mm}$ based on sketches of the cross-section of human SC (Paxinos and Mai, 2004). To assess the spatial spread of these response profiles, we also calculated their FWHM, as defined by their second spatial moment: $w=2 \sqrt{2 \ln 2 \int_{0}^{d_{m}}(x-c)^{2} A(s) d s / \int_{0}^{d_{m}} A(s) d s}$. Due to the presence of a substantial baseline response in the deep layers in the depth profiles, the first and second moments were calculated for profiles after subtracting the baseline level of activity, which was taken as the mean amplitude between depths $2.5-3 \mathrm{~mm}$ for each profile. Confidence intervals of the peak locations and centroids were also obtained by bootstrapping across runs 10,000 times.

We compared the measured depth profiles against a simple model composed of two layer-specific neural responses (Fig. 2) assumed to be constant over a particular depth range: a superficial component, amplitude $R_{s}$ in depth range $d_{1}-d_{2}$; and an intermediate component, amplitude $R_{i}$ in depth range $d_{3}-d_{4}$. The model also included a constant baseline, amplitude $B$, to reflect nonspecific or poorly localized components of the response over a separate range of $d_{0}-d_{5}=-0.4$ to $4 \mathrm{~mm}$. The model fMRI response was the sum of the two neural responses, $M(s)=R_{s}(s)+$
$R_{i}(s)$, convolved with an estimate of the fMRI point-spread function, chosen as a Gaussian with an FWHM of $1.2 \mathrm{~mm}$, plus the baseline $B(s)$. To obtain the depth parameters, we first estimated the thickness of the superficial and intermediate SC laminae based on published crosssectional sketches (Paxinos and Mai, 2004), taking into account the polar angle and eccentricity extent of the stimulus and our previous knowledge of SC retinotopy (Schneider and Kastner, 2005; Katyal et al., 2010). Due to the variability in depths of the SC laminae, we then adjusted the depth parameters $d_{1-4}$ together with $R_{\mathrm{s}}, R_{i}$, and $B$ at a coarse resolution to fit the model to the two depth profiles, minimizing the summed squared error between the model and the two profiles. This process yielded $d_{1}-d_{2}=$ $0-0.4 \mathrm{~mm}$ for the superficial gray layer, and $d_{3}-d_{4}=0.7-1.2 \mathrm{~mm}$ for the intermediate gray layer. Using these depth parameters values, we then finely sampled $R_{s}, R_{i}$, and $B$ between $0.01-1$ amplitude units to obtain the best fit of the model to our mean depth profiles. To obtain CIs on the amplitude parameters, we also fit each bootstrapped depth profile (normalized to unity), and this ensemble of fitted amplitude values was used to calculate their CIs.

We used these model fits to test two hypotheses. First, that the superficial response $R_{s}$ was reduced during the detection experiment as compared with discrimination. Second, we calculated the ratio of the superficial to intermediate layer response $\left(R_{s} / R_{i}\right)$ to test whether the ratio was greater for discrimination than detection.

\section{Results}

Threshold-contrast detection experiment

For the contrast detection paradigm, all five subjects performed multiple psychophysics sessions in a side-laboratory before scanning, where the contrast of the Gabor patches was continually varied to determine each subject's stable contrast-detection threshold $\left(d^{\prime} \sim 1\right)$. Threshold values of the luminance-contrast varied between $4.5-11 \%$ across subjects.

Inside the scanner, subjects performed the task while highresolution ( $1.2 \mathrm{~mm}$ isovoxel) fMRI data were obtained. The average behavioral performance across subjects during the scanning sessions was $69 \%$. Significant $(p<0.014)$ response amplitudes were observed within the ROIs for 7/10 colliculi and for all colliculi combined ( $p<10^{-4}$; Fig. $3 a$ ) at the temporal delay determined by the localizer sessions. The mean BOLD amplitude across all colliculi for the detection experiment was $0.25 \%$ compared with $0.66 \%$ for the localizer. When not restricting the data to the localizer-session hemodynamic delays, 9/10 colliculi had significant amplitudes $(p<0.04)$.

The location of the significant detection response on the SC surface (Fig. 4) corresponded well to the expected retinotopic locations (black outlines). We calculated the difference in the spatial centroids of the retinotopic ROIs and the detection response on the SC surface for each colliculus (Table 1). The mean difference in spatial centroids across colliculi was $0.8 \mathrm{~mm}$. When considering individual colliculi, centroid differences were small $(<1.2 \mathrm{~mm}$ ) for $9 / 10$ colliculi (Fig. 4; Table 1$)$.

Because SC responses are known to be primarily contralateral, we would expect that the block-alternation should produce a $12 \mathrm{~s}$ time delay between the responses of the two colliculi. Within the ROIs, the mean delay across all subjects was 9.5 s $(68 \%$ CIs: $4.8-14.2 \mathrm{~s}$; Table 1$)$. The nearly counter-phase character of the lateralized responses supports their correspondence to the cue alternation.

In Subject 1, we repeated the contrast detection session, to measure the repeatability of the spatial location of activation and intercollicular time delay. The data reproduced at very similar spatial locations (distance between the spatial centroids of activity between sessions: left colliculus, $0.91 \mathrm{~mm}$; right, $0.27 \mathrm{~mm}$ ) and similar delay (time-delay difference between sessions $=0.42 \mathrm{~s}$ ). 


\section{Threshold-contrast} control experiment

Three of the five subjects performed a control experiment to test whether the threshold-contrast Gabor detection targets evoked a measureable visual response within the retinotopic SC ROIs. Control experiment amplitudes were not significantly $>0$ in all six individual colliculi $(p>0.15)$ and combined colliculi (mean, $-0.07 \%$; $p>0.88$; Fig. $3 b$ ). Contrast detection response amplitudes were significantly greater than control experiment amplitudes in $3 / 6$ colliculi $(p<0.012)$.

\section{Speed discrimination experiment}

In this experiment, subjects viewed a visual stimulus that remained presented bilaterally and consistently. The only variation was a subtle cue that directed attention alternately to the left and right hemifields. This task evoked response amplitudes that were significantly $>0(p<0.004)$ in $6 / 10$ individual colliculi with a substantial trend $(p<0.084)$ in two more colliculi. Responses were very significant for all colliculi combined (mean, $0.31 \% ; p<10^{-4}$ ). The data were well lateralized across subjects with a mean delay between colliculi of $12.3 \mathrm{~s}$ (68\% CIs; 9.2-15.8 s).

\section{Depth profiles of activity}

Figure $5 a$ shows a plot of the amplitude of the BOLD response versus depth in the left colliculus of Subject 4 for two conditions: threshold-contrast detection (blue), and speed discrimination (red). Depth is defined as the distance (in $\mathrm{mm}$ ) from the interface of CSF and SC tissue (gray regions mark depths $<0$ ). Dotted lines show $68 \%$ CIs. This single colliculus profile shows that the response evoked by the discrimination task is closer to the SC surface than the detection task. When the depth profiles were averaged over both colliculi of all subjects (Fig. 5b), there was a clear separation in depth between the two profiles. We calculated the location of the peak of the profiles and centroids over $0-2.5$ $\mathrm{mm}$ in depth. The peak location of the detection response was $0.49 \mathrm{~mm}$ deeper $(p<0.015)$ than for the discrimination response, whereas the centroid of the detection response profile was deeper by $0.27 \mathrm{~mm}$ (marginally significant at $p<0.06$ ) than the discrimination profiles. Similar measures were also made over the depth range $0-3 \mathrm{~mm}$, for which peaks were still separated by $0.5 \mathrm{~mm}(p<0.02)$ and centroids separated by $0.27 \mathrm{~mm}$ $(p<0.1)$. The FWHM (second moment) of the profiles was 1.4 $\mathrm{mm}$ for both experiments. There was also a substantial positive baseline of the profiles, with $>50 \%$ of the activity extending deeply into the SC in both cases.

Figure $5 c$ shows the fit of our laminar neural response model to the normalized depth profiles. The fits explained 99 and $97 \%$ of the variance respectively for the discrimination and detection profiles. The mean values of $R_{s}, R_{i}$, and $B$ were $0.77,0.45$, and 0.55 , respectively, for the discrimination experiment, and 0.19 , 0.72 , and 0.62 , for the detection experiment. The superficial layer response for discrimination was significantly greater than for detection $(p<0.05)$. The intermediate layer response was greater for detection than discrimination, but this difference was not significant $(p>0.20)$. The combination of the two effects, which can be measured as the ratio of the superficial to intermediate layer response was also greater for discrimination than detection $(p<0.04)$ indicating a significant shift of the detection response deeper into the colliculus.

\section{Eye movements}

All five subjects were experienced psychophysics observers; all but one had $>50 \mathrm{~h}$ of experience performing tasks involving maintenance of fixation. To assure that subjects could perform the contrast detection task while maintaining fixation, we tracked eye-movements using an ASL Eye-Trac 6000 (Applied Science Laboratory) in three subjects while they performed the detection paradigm over six runs, outside the scanner. Subjects generally held fixation, but did make infrequent $(2.2 / \mathrm{min})$ saccades (deviations from fixation $>1^{\circ}$ ) with a mean length of $2.5^{\circ}$. These saccades typically fell well short of the stimulus aperture $\left(4^{\circ}-7 \cdot 5^{\circ}\right)$. Only $7 \%$ of the total saccades terminated within the stimulus aperture. To determine whether the saccades had a bias toward the cued hemifield, we treated the horizontal component of the saccades along the cued side as positive and along the opposite side as negative. The mean of this signed horizontal component along the cued hemifield in the three subjects was $-0.03^{\circ}, 0.08^{\circ}$, and $0.33^{\circ}$, which was significantly different from zero in only the third subject $(p<0.003)$ and the three subjects combined $(p<$ 0.005 ). Subject 3 , who showed the greatest tendency to make these infrequent saccades along the direction of the cued hemifield, was also the least experienced psychophysical observer. Because the fMRI response is both slow and population weighted (Heeger and Ress, 2002), these infrequent saccades can be expected to have little effect. We conclude that subjects can have a very slight tendency to make eye movements toward the cued hemifield, but this tendency is too small to affect our results.

\section{Discussion}

Our data show that SC has a reliably lateralized detection response, a putative correlate of endogenous attention. The detection paradigm sought to minimize the exogenous stimulus drive by using threshold-contrast stimuli, and counterbalancing their appearance across the two hemifields. Despite this effort, our use of a blocked paradigm did not permit separation of the responses evoked by the stimulus-present and -absent trials, so the faint Gabor stimuli could have evoked an exogenous response. However, the lack of significant visual drive observed in the control experiment leads us to conclude that the observed responses were dominated by top-down signals such as endogenous attention and was similar to the base response of observed in early visual cortex fMRI experiments (Kastner et al., 1999; Ress et al., 2000).

The spatial location of the detection response generally corresponded well to the independently localized ROIs. On 6/10 col- 


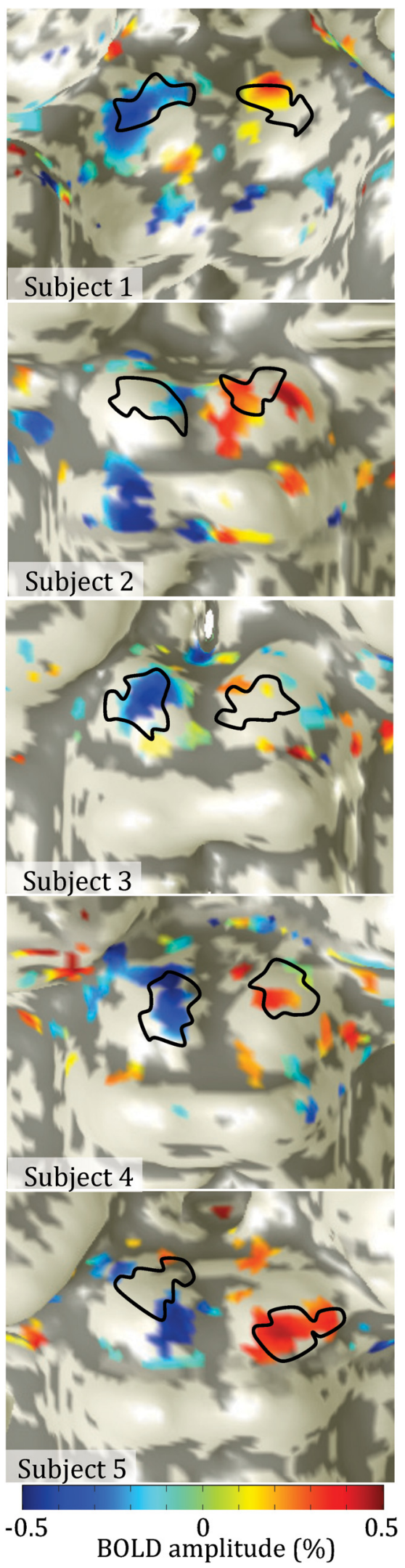

Figure 4. Significant $(p<0.05)$ detection-response shown on each subject's colliculi; black outlines show retinotopic ROIs. Colors show amplitude of BOLD response with positive amplitudes expected to occur on the right colliculus and negative amplitudes on the left colliculus.
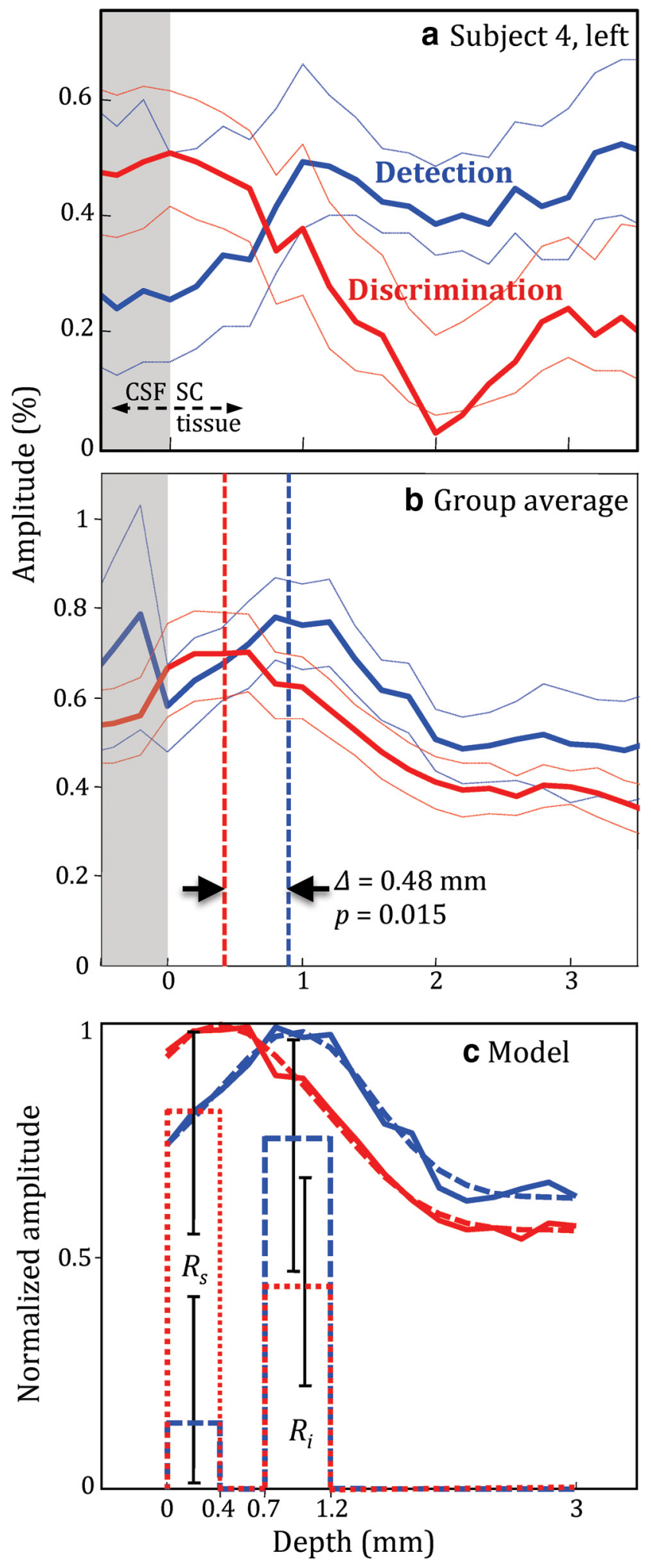

Figure 5. Depth profiles obtained from retinotopic ROIs. $\boldsymbol{a}$, Amplitude versus depth for detection (blue) and discrimination (red) response in Subject 4. Dotted lines are $68 \%$ Cls. $\boldsymbol{b}$, Profiles averaged over both colliculi of all subjects. $c$, Normalized average profiles (solid) along with the model fits (dashed) and responses $R_{s^{\prime}} R_{i}$ (same color scheme as profiles) with $68 \% \mathrm{Cls}$.

liculi, there was extremely good correspondence (centroid offsets $<0.7 \mathrm{~mm}$ ) between the detection response and the retinotopic ROIs. In a few of the colliculi, there was some mislocalization. There are several possible causes for the observed mismatch, in- 
cluding misalignment between the functional data and the reference volume, and limited hemodynamic specificity. There are also small regions of apparently significant activity on other portions of the midbrain, particularly on dorsal portions of the inferior colliculus in Subjects 1 and 2. This activity could be spurious, the consequence of contamination from superficial vascular structures. Nonetheless, the generally good spatial correspondence $(0.8 \mathrm{~mm}$ mean centroid separation) demonstrated the ability of our high-resolution fMRI techniques (Katyal et al., 2012) to perform retinotopically localized experiments within the human SC with $<1 \mathrm{~mm}$ accuracy.

The detection response was temporally correlated with the lateralized task alternation. However, there was some variability in the intercollicular delay across subjects. Such variability could reflect at least two mechanisms. First, subjects may exhibit differences in their ability to deploy endogenous attention between the two hemifields. On one side or the other, they may be able to more rapidly deploy their attention during each block. Second, the effect could be vascular, possibly reflecting differences in hemodynamic delays between the two colliculi. However, the former hypothesis is supported by the much more precisely counterphase time delays observed in the discrimination experiment.

The depth profiles strongly suggest that the detection and discrimination tasks evoke different depth profiles of activity in SC (Fig. 5). Because of the limited spatial specificity of the BOLD response, we obtained spatially broad response distribution with a FWHM of $1.4 \mathrm{~mm}$ in both conditions. This is consistent with the tissue-oxygen measurements of Thompson et al. (2005), who reported that the blood-flow response evoked by visual stimulation had a $1.4 \mathrm{~mm}$ FWHM in the lateral geniculate nucleus (LGN). In cortex, on the other hand, the point-spread function of BOLD has been measured to be close to $3 \mathrm{~mm}$ (Engel et al., 1997; Parkes et al., 2005), but these measurements were carried using coarse spatial resolution. Using much finer sampling $(0.3 \times$ $0.3 \times 2 \mathrm{~mm}$ voxels) in cats, however, Duong et al. (2001) measured a $0.5 \mathrm{~mm}$ FWHM using cerebral blood flow and BOLD MRI at 4.7 T. In our case, the FWHM resolution would be limited by our voxel sampling of $\sim 1.2 \mathrm{~mm}$ as well as the depth extent of the neural response. The deep $(>2.5 \mathrm{~mm})$ baseline level of activity in both the profiles may reflect a top-down response in all layers that is similarly driven by both the contrast detection and discrimination tasks. Alternatively, this response could also be a consequence of the limited spatial specificity of the BOLD response and the sampling resolution of our measurements. Nevertheless, the significant shift of the detection profiles deeper into the colliculus compared with the discrimination profile very likely indicates a correspondingly deeper neural response profile.

The detection-response activity peaks more deeply within the tissue of the colliculus $(\sim 0.9 \mathrm{~mm})$ compared with the discrimination response $(\sim 0.4 \mathrm{~mm})$. Based on anatomical and neurophysiological studies in primates, we propose that these differences correspond to the laminar distribution of neuronal subtypes within SC (Goldberg and Wurtz, 1972; Wurtz and Mohler, 1976; Ignashchenkova et al., 2004). The more superficial response for discrimination would correspond to an enhanced activity of both the superficial-layer visual and intermediate-layer visuomotor neurons, whereas the deeper detection response would primarily correspond to the cue-evoked baseline enhancement of the visuomotor neurons (Ignashchenkova et al., 2004). A simple laminar neural response model, which provided excellent fits to the response profiles supported this hypothesis, showing that discrimination evoked a significantly greater response in the superficial layers as compared with detection.
Our results support a role for the intermediate layers of SC in the mediation of endogenous attention. These findings complement recent studies in macaques that suggest a critical role for SC in endogenous attention. Reversible inactivation of SC in macaques induces behavioral deficits similar to those observed in human attentional neglect (Lovejoy and Krauzlis, 2010; Zénon and Krauzlis, 2012). Also, subthreshold microstimulation of the SC intermediate layers improves behavioral performance in a spatially selective manner that is similar to endogenous attention (Müller et al., 2005; Cavanaugh et al., 2006). However, the same study that observed a baseline increase in visuomotor single-unit activity for attention, showed that it occurred specifically for peripheral and not central cues, suggesting that enhancement of visuomotor neuronal response in SC is a correlate of exogenous and not endogenous attention (Ignashchenkova et al., 2004).

The endogenous attentional response that we observed using fMRI may not have been directly evident to monkey single-unit electrophysiology measurements for several reasons. First, it is possible that effects of endogenous attention upon visuomotor neurons are weak, and therefore only evident to a populationaveraged metric such as fMRI. The fMRI response has been shown to be better correlated with local field potentials changes, reflecting subthreshold changes in network processing, rather than single-unit spiking activity (Logothetis et al., 2001; Angenstein et al., 2009). Alternatively, the fMRI base response in SC may reflect subtler variations of the neural dynamics rather than increased firing rate, such as reduction of spike-rate variability (Mitchell et al., 2007), or interneuronal correlations (Cohen and Maunsell, 2009). These variations could give rise to metabolic changes in the neurons that give rise to a blood-flow response and a BOLD signal.

The deeper response that we observed for attention in the absence of a visual stimulus, together with monkey electrophysiology results (Kustov and Robinson, 1996; Ignashchenkova et al., 2004; Müller et al., 2005), supports an oculomotor theory of attention (Rizzolatti et al., 1987; Corbetta et al., 1998; Moore et al., 2003). According to this theory, the neural substrates for visual spatial attention overlap with those that mediate the preparation for eye movements. The overt eye movement then follows this preparation process.

However, there are other top-down signals that could correspond to our observed detection response aside from attention. For example, the task may encourage subjects to consider performing orienting head movements toward the cued sectors, and these intentions could give rise to these signals. The deeper layers of SC are involved in both eye movements and head movements (Cowie and Robinson, 1994; Freedman et al., 1996). We cannot rule out the possibility that the detection response corresponds to a suppressed intention to perform such orienting behavior. Additionally, the task may have modulated subjects' fixational eye movements. SC contains neurons responsive to microsaccades near the foveal (rostral) regions (Hafed et al., 2009), and recent evidence has suggested a link between microsaccades and covert attention (Engbert and Kliegl, 2003; Hafed et al., 2009). However, in our experiment the observed responses were in more central regions of SC that correspond to the peripheral visual field, reducing the likelihood that the responses were generated by microsaccade-related activity.

In monkey SC, two classes of saccade-related intermediate layer neurons have been distinguished: "buildup" neurons that exhibit a gradual increase of activity before saccades, and "burst" neurons that fire at saccade onset. For overt orienting behavior, these neurons operate together to induce saccadic eye move- 
ments (Munoz and Wurtz, 1995; Freedman and Sparks, 1997) Recent work in monkeys has shown that in addition to sending eye movement signals to the brainstem oculomotor nuclei, the eye-movement generation signal are also projected upstream from SC intermediate layers to cortex as a corollary discharge (Sommer and Wurtz, 2002, 2004).

The visuomotor neurons showing baseline response enhancement for attention have properties similar to the buildup neurons (Ignashchenkova et al., 2004). Motivated by the oculomotor theory, we speculate that in the absence of overt behavior, these neurons accumulate evidence for orienting covert spatial attention by combining cognitive and multisensory inputs from other brain regions such as frontal eye fields and association cortices (Sommer and Wurtz, 2000; May, 2006). Moreover, the attention signals from these neurons could then modulate other subcortical and cortical regions, making SC part of a subcortical branch of the top-down attention network, in addition to the more generally accepted cortical network (Corbetta and Shulman, 2002). Such a network of attention has been suggested previously (Kastner and Pinsk, 2004; Wurtz et al., 2011; Krauzlis et al., 2013) and could also involve multiple thalamic nuclei that are also known to be modulated by attention such as, pulvinar, LGN, and the thalamic reticular nucleus (Robinson and Petersen, 1992; Kastner et al., 2004; McAlonan et al., 2008). However, more work is required to understand the detailed dynamics of attentional signals within subcortical structures and their relationship with cortex.

\section{References}

Angenstein F, Kammerer E, Scheich H (2009) The BOLD response in the rat hippocampus depends rather on local processing of signals than on the input or output activity: a combined functional MRI and electrophysiological study. J Neurosci 29:2428-2439. CrossRef Medline

Biswal B, Yetkin FZ, Haughton VM, Hyde JS (1995) Functional connectivity in the motor cortex of resting human brain using echo-planar MRI. Magn Reson Med 34:537-541. CrossRef Medline

Brainard DH (1997) The psychophysics toolbox. Spat Vis 10:433-436. CrossRef Medline

Cavanaugh J, Wurtz RH (2004) Subcortical modulation of attention counters change blindness. J Neurosci 24:11236-11243. CrossRef Medline

Cavanaugh J, Alvarez BD, Wurtz RH (2006) Enhanced performance with brain stimulation: attentional shift or visual cue? J Neurosci 26:1134711358. CrossRef Medline

Cohen MR, Maunsell JH (2009) Attention improves performance primarily by reducing interneuronal correlations. Nat Neurosci 12:1594-1600. CrossRef Medline

Corbetta M, Shulman GL (2002) Control of goal-directed and stimulusdriven attention in the brain. Nat Rev Neurosci 3:201-215. CrossRef Medline

Corbetta M, Akbudak E, Conturo TE, Snyder AZ, Ollinger JM, Drury HA, Linenweber MR, Petersen SE, Raichle ME, Van Essen DC, Shulman GL (1998) A common network of functional areas for attention and eye movements. Neuron 21:761-773. CrossRef Medline

Cowie RJ, Robinson DL (1994) Subcortical contributions to head movements in macaques: I. Contrasting effects of electrical stimulation of a medial pontomedullary region and the superior colliculus. J Neurophysiol 72:2648-2664. Medline

Duong TQ, Kim DS, Uğurbil K, Kim SG (2001) Localized cerebral blood flow response at submillimeter columnar resolution. Proc Natl Acad Sci U S A 98:10904-10909. CrossRef Medline

Engbert R, Kliegl R (2003) Microsaccades uncover the orientation of covert attention. Vis Res 43:1035-1045. CrossRef Medline

Engel SA, Glover GH, Wandell BA (1997) Retinotopic organization in human visual cortex and the spatial precision of functional MRI. Cereb Cortex 7:181-192. CrossRef Medline

Fecteau JH, Bell AH, Munoz DP (2004) Neural correlates of the automatic and goal-driven biases in orienting spatial attention. J Neurophysiol 92: 1728-1737. CrossRef Medline

Freedman EG, Sparks DL (1997) Activity of cells in the deeper layers of the superior colliculus of the rhesus monkey: evidence for a gaze displacement command. J Neurophysiol 78:1669-1690. Medline

Freedman EG, Stanford TR, Sparks DL (1996) Combined eye-head gaze shifts produced by electrical stimulation of the superior colliculus in rhesus monkeys. J Neurophysiol 76:927-952. Medline

Friston KJ, Josephs O, Zarahn E, Holmes AP, Rouquette S, Poline J (2000) To smooth or not to smooth? Bias and efficiency in fMRI time-series analysis. Neuroimage 12:196-208. CrossRef Medline

Glover GH, Lai S (1998) Self-navigated spiral fMRI: interleaved versus single-shot. Magn Reson Med 39:361-368. CrossRef Medline

Glover GH, Li TQ, Ress D (2000) Image-based method for retrospective correction of physiological motion effects in fMRI: RETROICOR. Magn Reson Med 44:162-167. CrossRef Medline

Goldberg ME, Wurtz RH (1972) Activity of superior colliculus in behaving monkey: I. Visual receptive fields of single neurons. J Neurophysiol 35: 542-559. Medline

Hafed ZM, Goffart L, Krauzlis RJ (2009) A neural mechanism for microsaccade generation in the primate superior colliculus. Science 323:940-943. CrossRef Medline

Heeger DJ, Ress D (2002) What does fMRI tell us about neuronal activity? Nat Rev Neurosci 3:142-151. CrossRef Medline

Holmes A, Josephs O, Buchel C, Friston K (1997) Statistical modelling of low-frequency confounds in fMRI. Neuroimage 5:S480.

Ignashchenkova A, Dicke PW, Haarmeier T, Thier P (2004) Neuronspecific contribution of the superior colliculus to overt and covert shifts of attention. Nat Neurosci 7:56-64. CrossRef Medline

Kastner S, Pinsk MA (2004) Visual attention as a multilevel selection process. Cogn Affect Behav Neurosci 4:483-500. CrossRef Medline

Kastner S, Pinsk MA, De Weerd P, Desimone R, Ungerleider LG (1999) Increased activity in human visual cortex during directed attention in the absence of visual stimulation. Neuron 22:751-761. CrossRef Medline

Kastner S, O'Connor DH, Fukui MM, Fehd HM, Herwig U, Pinsk MA (2004) Functional imaging of the human lateral geniculate nucleus and pulvinar. J Neurophysiol 91:438-448. CrossRef Medline

Katyal S, Zughni S, Greene C, Ress D (2010) Topography of covert visual attention in human superior colliculus. J Neurophysiol 104:3074-3083. CrossRef Medline

Katyal S, Greene C, Ress D (2012) High-resolution functional magnetic resonance imaging methods for human midbrain. J Vis Exp 10:e3746. CrossRef Medline

Khan R, Zhang Q, Darayan S, Dhandapani S, Katyal S, Greene C, Bajaj C, Ress D (2011) Surface-based analysis methods for high-resolution functional magnetic resonance imaging. Graph Models 73:313-322. CrossRef Medline

Krauzlis RJ, Lovejoy LP, Zénon A (2013) Superior colliculus and visual spatial attention. Annu Rev Neurosci 36:165-182. CrossRef Medline

Krüger G, Glover GH (2001) Physiological noise in oxygenation-sensitive magnetic resonance imaging. Magn Reson Med 46:631-637. CrossRef Medline

Kustov AA, Robinson DL (1996) Shared neural control of attentional shifts and eye movements. Nature 384:74-77. CrossRef Medline

Logothetis NK, Pauls J, Augath M, Trinath T, Oeltermann A (2001) Neurophysiological investigation of the basis of the fMRI signal. Nature 412: 150-157. CrossRef Medline

Lovejoy LP, Krauzlis RJ (2010) Inactivation of primate superior colliculus impairs covert selection of signals for perceptual judgments. Nat Neurosci 13:261-266. CrossRef Medline

May PJ (2006) The mammalian superior colliculus: laminar structure and connections. Prog Brain Res 151:321-378. CrossRef Medline

McAlonan K, Cavanaugh J, Wurtz RH (2008) Guarding the gateway to cortex with attention in visual thalamus. Nature 456:391-394. CrossRef Medline

Mitchell JF, Sundberg KA, Reynolds JH (2007) Differential attentiondependent response modulation across cell classes in macaque visual area V4. Neuron 55:131-141. CrossRef Medline

Moore T, Armstrong KM, Fallah M (2003) Visuomotor origins of covert spatial attention. Neuron 40:671-683. CrossRef Medline

Müller JR, Philiastides MG, Newsome WT (2005) Microstimulation of the superior colliculus focuses attention without moving the eyes. Proc Natl Acad Sci U S A 102:524-529. CrossRef Medline

Munoz DP, Wurtz RH (1995) Saccade-related activity in monkey superior 
colliculus: I. Characteristics of burst and buildup cells. J Neurophysiol 73:2313-2333. Medline

Nestares O, Heeger DJ (2000) Robust multiresolution alignment of MRI brain volumes. Magn Reson Med 43:705-715. CrossRef Medline

Parkes LM, Schwarzbach JV, Bouts AA, Deckers RH, Pullens P, Kerskens CM, Norris DG (2005) Quantifying the spatial resolution of the gradient echo and spin echo BOLD response at 3 Tesla. Magn Reson Med 54:14651472. CrossRef Medline

Paxinos G, Mai JK (2004) The human nervous system, Ed 2. New York: Academic.

Pelli DG (1997) The VideoToolbox software for visual psychophysics: transforming numbers into movies. Spat Vis 10:437-442. CrossRef Medline

Ress D, Backus BT, Heeger DJ (2000) Activity in primary visual cortex predicts performance in a visual detection task. Nat Neurosci 3:940-945. CrossRef Medline

Ress D, Glover GH, Liu J, Wandell B (2007) Laminar profiles of functional activity in the human brain. Neuroimage 34:74-84. CrossRef Medline

Rizzolatti G, Riggio L, Dascola I, Umiltá C (1987) Reorienting attention across the horizontal and vertical meridians: evidence in favor of a premotor theory of attention. Neuropsychologia 25:31-40. CrossRef Medline

Robinson DL, Petersen SE (1992) The pulvinar and visual salience. Trends Neurosci 15:127-132. CrossRef Medline

Schneider KA, Kastner S (2005) Visual responses of the human superior colliculus: a high-resolution functional magnetic resonance imaging study. J Neurophysiol 94:2491-2503. CrossRef Medline
Sommer MA, Wurtz RH (2000) Composition and topographic organization of signals sent from the frontal eye field to the superior colliculus. J Neurophysiol 83:1979-2001. Medline

Sommer MA, Wurtz RH (2002) A pathway in primate brain for internal monitoring of movements. Science 296:1480-1482. CrossRef Medline

Sommer MA, Wurtz RH (2004) What the brain stem tells the frontal cortex: I. Oculomotor signals sent from superior colliculus to frontal eye field via mediodorsal thalamus. J Neurophysiol 91:1381-1402. CrossRef Medline

Thompson JK, Peterson MR, Freeman RD (2005) Separate spatial scales determine neural activity-dependent changes in tissue oxygen within central visual pathways. J Neurosci 25:9046-9058. CrossRef Medline

Worsley KJ, Friston KJ (1995) Analysis of fMRI time-series revisited: again. Neuroimage 2:173-181. CrossRef Medline

Wurtz RH, Mohler CW (1976) Organization of monkey superior colliculus: enhanced visual response of superficial layer cells. J Neurophysiol 39:745765. Medline

Wurtz RH, McAlonan K, Cavanaugh J, Berman RA (2011) Thalamic pathways for active vision. Trends Cogn Sci 15:177-184. CrossRef Medline

Xu G, Pan Q, Bajaj CL (2006) Discrete surface modeling using partial differential equations. Comput Aided Geom Des 23:125-145. CrossRef Medline

Yushkevich PA, Piven J, Hazlett HC, Smith RG, Ho S, Gee JC, Gerig G (2006) User-guided 3D active contour segmentation of anatomical structures: significantly improved efficiency and reliability. Neuroimage 31:11161128. CrossRef Medline

Zénon A, Krauzlis RJ (2012) Attention deficits without cortical neuronal deficits. Nature 489:434-437. CrossRef Medline 